\title{
Computational Fluid Dynamics Modeling and Investigation of Horizontal Airflow Ventilation System in Surgical Site in Hospital
}

\author{
S B Thool ${ }^{1}$, S L Sinha ${ }^{2}$ \\ Department of Mechanical Engineering \\ ${ }^{I}$ Rungta College of Engineering and Technology, Bhilai, India \\ ${ }^{2}$ National Institute of Technology, Raipur, India
}

\begin{abstract}
The present paper deals with the numerical study of horizontal laminar flow ventilation system for surgical site in hospital. When the orientation of supplies openings and exhausts are altered, the performance of ventilation system in terms of effectiveness of removal of contaminated particles is also affected. In this investigation, the performance of new horizontal Single Sided Supply and Exhaust (SSSE) ventilation system have been evaluated and compared withconventional Vertical Unidirectional (Laminar) ventilation system using Computational Fluid Dynamics (CFD) technique.

It has beenobserved that contaminant control is found more effective in the case of SSSE horizontal ventilating system over the Vertical Unidirectional ventilation system. It is so happened because the airflow pattern over the critical area of surgery is not affected by the overhead obstacle i.e., surgical lamp. Investigation also reveals that the thermal plume induced by the surgical wound and the surgical lamp, is not counteracted by the supply airflow pattern, which plays an important roll in positive way.
\end{abstract}

Keywords: Ventilation system, Airflow pattern, Infection control, surgical site infection, CFD, Single Sided Supply and Exhaust (SSSE) ventilation system.

\section{Introduction}

In order to control the Surgical Site Infection (SSI) caused by airborne particles, application of ventilation systems is well known. Infectious particles can be effectively isolated, diluted and get escaped from surgical site through the proper distribution of ultraclean air [1]. Now a days, downwardunidirectional (laminar) flow [2] is widely adopted air distribution for creating an aseptic environment around patient [3,4].

Even though the downward unidirectional airflow pattern is recommended as a regular design, current research demonstrates some disadvantages. Overhead accessories such as medical lamps can easily affect the unidirectional airflow pattern of downward airflow and thermal plume around the wound. [5,6]. Though some methods to reduce these negative effects have been recommended adequately, some disadvantages cannot be eliminated due to the vertical unidirectional flow pattern [7]. The main influencing factor for airflow pattern is to consider the indoor obstacles, thus counteracting the function of clean air in isolating infectious particles. Facilities placed upstream of patient such as a medical lamp may cause serious whirlpool and particulate accumulation [8]. Further, the temperature of human body surface is usually higher than the one of his surrounding air, whichcauses thebuoyancy driven upward airflow plumes. This can easily disturb the downward airflow pattern formed by the ventilating system and carry infectious particles to the wound, leading to serious surgical site infection.

To overcome the disadvantages of the conventional airflow pattern applied to operating room, an alternative of horizontal airflow pattern should be studied to find out its feasibility, characteristics and contamination control effect in operating room. The main sources of infectious particles and bacteria in an operating room are the surgeons relatedto their activities. Isolating patient from surgeon can effectively prevent SSI by formation of proper airflow pattern. A horizontal airflow with single sided supply and exhaust ventilation system supplying ultraclean air will avoid the obstacles, such as medical lamps. Again the horizontal airflow with a direction perpendicular to the airflow direction of thermal plume around surgeon and patient can avoid direct counteractive effect between the two airflows.

The existing design of horizontal airflow system i.e. opposite sided supply and exhaust ventilation system causes a relatively long air path length. It makes it difficult to maintain a washing effect around the patient with unidirectional flow. At most time the surgical staff and necessary furniture are serious obstacles to damage the unidirectional airflow [9].

Investigating and comparing above stated ventilation system experimentally, is very costly and time consuming. Due to technological and economical difficulties found when performing and comparing experiments, numerical studies of the problem appears to be a promising method for quantifying the effect of the various parameters that influence the design of best ventilation system. 


\subsection{Airflow model}

\section{CFD MODELING}

In this investigation, the air is treated as continuum moving in the room as governed by the Eulerian conservation equation (Patankar, 1980) [10].

$\frac{\partial}{\partial t}(\rho \phi)+\operatorname{div}(\rho V \phi)=\operatorname{div}\left(\Gamma_{\phi} \operatorname{grad} \phi\right)+S_{\phi}$

where, $\phi$ represents the independent variables: time averaged velocity components $V$ (i.e. $u, v, w$ ), turbulent kinetic energy, $k$, dissipation rate of turbulent kinetic energy, $\varepsilon$, and enthalpy $H$. When $\phi$ is unity, the equation represents the conservation of mass. Expressions for the effective diffusivity, $\Gamma_{\phi}$ and source term, $S_{\phi}$ for each variable and the corresponding empirical numbers are described by Launder and Spalding (1972) [11].

The fluid motion in most cases of ventilation system does not hold a well-structured behavior in terms of laminar flow. It consist of free jets near the supply air outlet with entrainment and shear layers, attachment and recirculation zones, boundary layers along the walls. In reality, at reasonable ventilation rates, the flow is fully turbulent in the supply air ducts, HVAC outlets / inlets and downstream of the edges of the obstacles. In order to model the random feature of turbulent flows, a time decomposition (also called Reynolds decomposition) of the instantaneous flow variables $\phi(t)$ is introduced into the governing flows.

$\phi(t)=\bar{\phi}+\phi^{\prime}(t)$

The mean value of $\phi(t)$ is obtained by integrating $\phi(t)$ over a period of time $d t$ that is much longer than the fluctuating duration:

$\bar{\phi}=\frac{1}{\tau} \int_{t}^{t+d t} \phi\left(t_{1}\right) d t_{1}$

Using "Reynolds rules", all instantaneous flow equations are time averaged. In this case, time averaging will be presented by considering a three-dimensional flow ( $\mathrm{x}, \mathrm{y}$ and $\mathrm{z}$ directions) located in a gravity field opposed to y direction and assuming flow to be Newtonian, incompressible under non-isothermal condition

Boussinesq hypothesis is employed, which neglects the variations of physical characteristics of the fluid in all equations, except for the density in the buoyancy term of the vertical momentum conservation equation (the density variations in the buoyancy term induce thevertical motion of the fluid in natural convection). Thus the various terms of themomentum conservation equations could be divided by the density, $\rho$ of the fluid in reference conditions.

\subsection{Equations for particle motion and dynamics}

Discrete phase particle model based on the Lagrangian approach is used to calculate the particle trajectories. Particle motion in carrier fluid is affected by various forces such as viscous drag force, gravity force, added mass force (virtual mass force), Brownian force, and pressure force. In this study, Brownian force has been ignored due to the large size of particle. The added mass force was considered in a few simulations and was found to have negligible influence on particle trajectory [12]. Thus in this study, steady viscous drag force, gravity force and pressure force have been considered.

The individual trajectory is calculated by solving the momentum equation. By equating the particle inertia with external forces, the momentum equations can be expressed as:

$$
\begin{aligned}
& m_{p} \frac{d u_{p}}{d t}=\frac{1}{2} C_{D} A_{p} \rho\left(u-u_{p}\right) \sqrt{\left(u-u_{p}\right)^{2}+\left(v-v_{p}\right)^{2}+\left(w-w_{p}\right)^{2}}+m_{p} g_{x} \\
& m_{p} \frac{d v_{p}}{d t}=\frac{1}{2} C_{D} A_{p} \rho\left(v-v_{p}\right) \sqrt{\left(u-u_{p}\right)^{2}+\left(v-v_{p}\right)^{2}+\left(w-w_{p}\right)^{2}}+m_{p} g_{y} \\
& m_{p} \frac{d w_{p}}{d t}=\frac{1}{2} C_{D} A_{p} \rho\left(w-w_{p}\right) \sqrt{\left(u-u_{p}\right)^{2}+\left(v-v_{p}\right)^{2}+\left(w-w_{p}\right)^{2}}+m_{p} g_{z}
\end{aligned}
$$

where

$u, v, w \quad-\quad$ instantaneous velocity of air in $\mathrm{x}, \mathrm{y}$ and $\mathrm{z}$ directions; $u_{p}, v_{p}, w_{p} \quad-\quad$ particle velocity in $\mathrm{x}, \mathrm{y}$ and $\mathrm{z}$ direction; 


$\begin{array}{lll}x_{p}, y_{p}, z_{p} & - & \text { movement of particle in } \mathrm{x}, \mathrm{y} \text { and } \mathrm{z} \text { direction; } \\ g_{x}, g_{y}, g_{z} & - & \text { acceleration due to gravity in } \mathrm{x}, \mathrm{y} \text { and } \mathrm{z} \text { directions; } \\ A_{p} & - & \text { cross-sectional area of the particle; } \\ m_{p} & - & \text { mass of particle; } \\ \rho & - & \text { density of the particle; } \\ C_{D} & - & \text { drag coefficient; } \\ d t & - & \text { time interval. }\end{array}$

where

$C_{D}=\frac{24}{R e}\left(1+\frac{3}{16} R e\right)^{0.5} \quad$ for $R e \leq 560$

and

$C_{D}=0.44 \ldots$ for $R e>560$

The Reynolds number of the particle is based on the relative velocity between particle and air.

In laminar flow, particles released from a point source with the same weight would initially follow the airstream in the same path and then fall under the effect of gravity. Unlike laminar flow, the random nature of turbulence indicates that the particles released from the same point source will be randomly affected by turbulent eddies. As a result, it will be diffused away from the streamline at different fluctuating levels. In order to model the turbulent diffusion, the instantaneous fluid velocities in the three Cartesian directions $\mathrm{u}, \mathrm{v}$ and $\mathrm{w}$ are decomposed into the mean velocity component and the turbulent fluctuating component as:

$u=\bar{u}+u^{\prime} ; \quad v=\bar{v}+v^{\prime} ; \quad w=\bar{w}+w^{\prime}$

where, $\bar{u}$ and $u$ ' are the mean and fluctuating components of velocity in $x$-direction. The same applies for $y$ and $z$ directions. The stochastic approach prescribes the use of a random number generator algorithm, which, in this case, is taken from Press et al. [13] to model the fluctuating velocity. It is achieved by using a random sampling of a Gaussian distribution with a mean of zero and a standard deviation of unity. Assuming isotropic turbulence, the instantaneous velocity of air are then calculated from kinetic energy of turbulence:

$u=\bar{u}+N \alpha ; \quad v=\bar{v}+N \alpha ; \quad w=\bar{w}+N \alpha$

Where $N$ is the pseudo-random number, ranging from 0 to 1 , with

$\alpha=\left(\frac{2 k}{3}\right)^{0.5}$

The mean velocity, which are the direct output of CFD, determine the convection of the particles along the streamline, while the turbulent fluctuating velocity, $N \alpha$, contributes to the turbulent diffusion of the particle.

\section{Validation \& Flow Visualization}

The validation of the pattern of airflow by CFD softwareis done by the flow visualization. The geometrical structure of the model room is constructed with width, depth and height of the room as $100 \mathrm{~cm}, 50$ $\mathrm{cm}$ and $38 \mathrm{~cm}$ respectively. Within the model room, a partition with a height of $19 \mathrm{~cm}$ is located in the middle of the room. Air is allowed to enter the model room through one ceiling inlet vent through duct and to leave through the other ceiling outlet vent.

Air along with the smoke produced from burning incense stick enters the test model room through the diffuser of airflow facility. Smoke follows the path of mainstream since its density is approximately same as that of air and it does disturb the airflow pattern appreciably. The photographs of the streamline pattern were taken for each test run. Reynolds number and Grashof number were calculated and results of the numerical simulation for three-dimensional, steady and laminar flow have been obtained. Streamlines contours (Fig. 1) obtained from numerical simulation and photograph of smoke flow (Fig. 2) have been found similar. The recirculating cells on either sides of the main stream have been seen by the naked eyes but could not be captured by the camera due to lack of sufficient amount of smoke in these regions. 

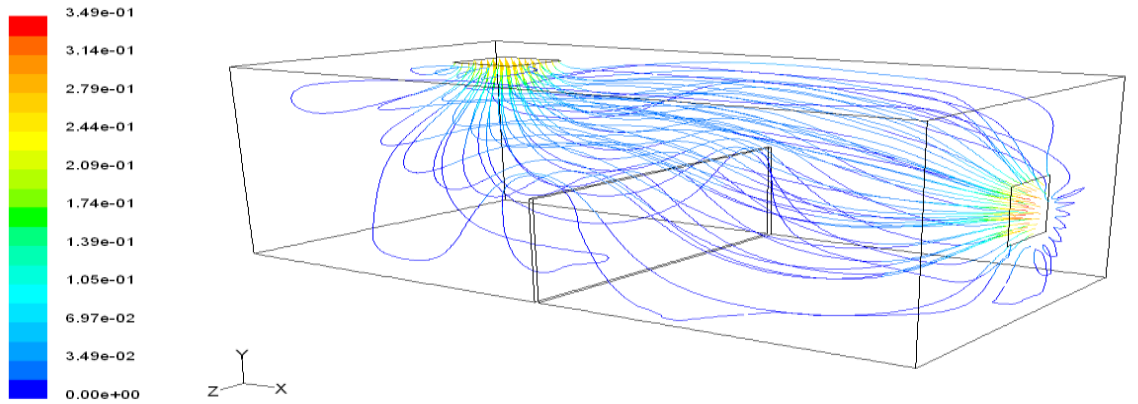

Fig. 1 Streamlines of air distribution in model room

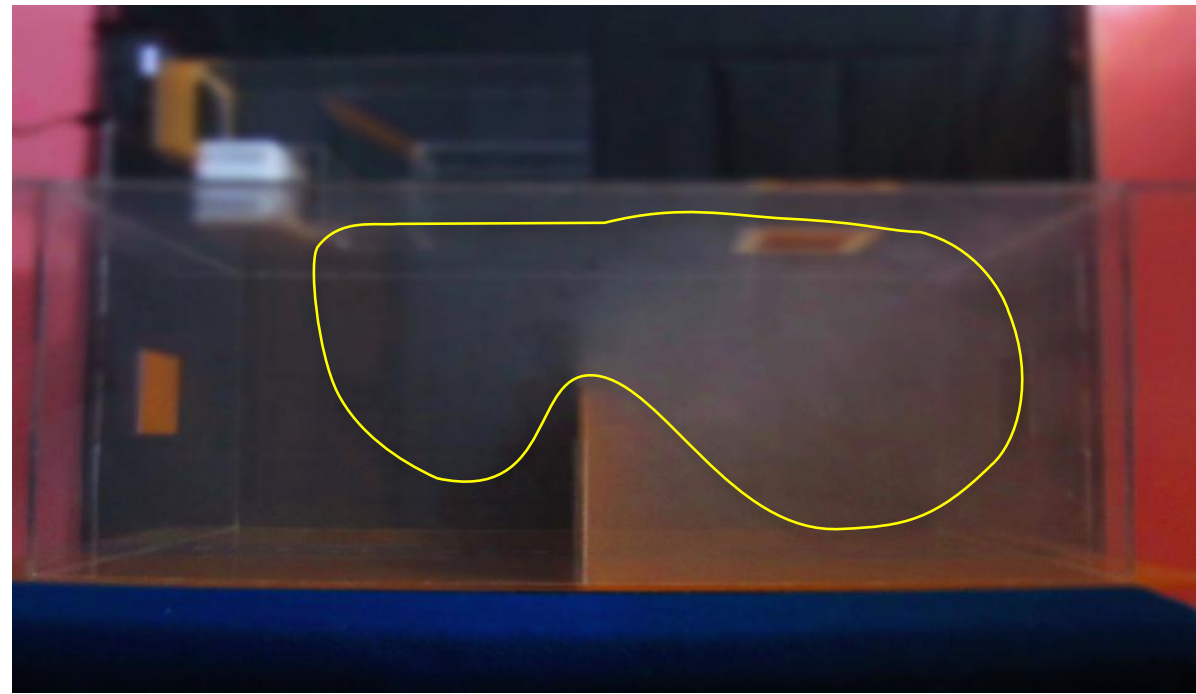

Fig. 2 Flow Visualization Using Smoke

\section{Brief Of Operating Room}

In a typical operating room layout,five surgical staff members, lights, machinery, tables and patient are considered for the baseline model for the CFD simulations. The brief description of operating room is given in the Fig. 3 and Table 1.The size of each inlet and exhaust grille is $0.61 \mathrm{~m} \times 0.36 \mathrm{~m}$. Surgical site (wound area) and back table is considered to be the critical area. The most suitable operating value of ACH for this system is ranging from $60 \mathrm{~h}^{-1}$ to $65 \mathrm{~h}^{-1}$. The present CFD simulation is done taking ACH as $63 \mathrm{~h}^{-1}$ and air velocity as 0.14 $\mathrm{m} / \mathrm{s}$ with temperature as $27 \mathrm{C}^{\circ}$.

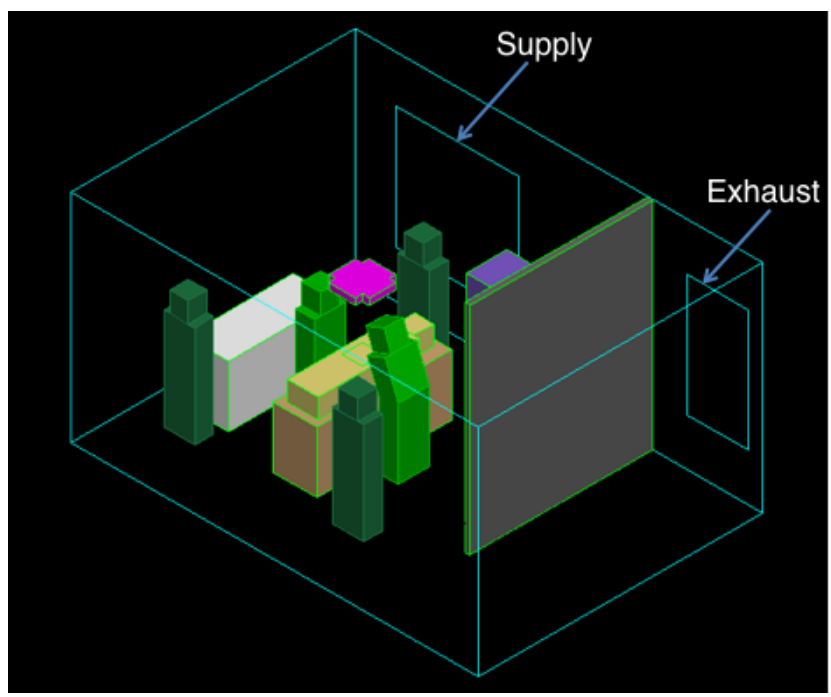

Fig. 3 Baseline model of the operating room 
Table 1

Dimension of operating room and other available items

\begin{tabular}{|l|l|}
\hline Item & Dimensions \\
\hline Operating table & $0.64 \mathrm{~m} \times 2.0 \mathrm{~m} \times 0.9 \mathrm{~m}$ \\
\hline Surgical lamp & $0.55 \mathrm{~m} \times 0.55 \mathrm{~m} \times 0.1 \mathrm{~m}$ \\
\hline Anesthesia machine & $0.6 \mathrm{~m} \times 0.6 \mathrm{~m} \times 1.1 \mathrm{~m}$ \\
\hline Back table & $0.64 \mathrm{~m} \times 1.6 \mathrm{~m} \times 0.9 \mathrm{~m}$ \\
\hline Monitor stand & $0.6 \mathrm{~m} \times 0.5 \mathrm{~m} \times 1.20 \mathrm{~m}$ \\
\hline Monitor & $0.5 \mathrm{~m} \times 0.4 \mathrm{~m} \times 0.6 \mathrm{~m}$ \\
\hline $\begin{array}{l}\text { Surgical staff (Two surgeon and } \\
\text { three nurses) }\end{array}$ & $0.46 \mathrm{~m} \times 0.28 \mathrm{~m} \times 1.8 \mathrm{~m}$ each \\
\hline Patient & $0.46 \mathrm{~m} \times 0.28 \mathrm{~m} \times 1.8 \mathrm{~m}$ \\
\hline
\end{tabular}

\section{Boundary Conditions}

The velocity, temperature and turbulent transport quantities over the inlet boundary are prescribed from the experimental data found by Memarzadeh and Manning [14]. Outlet boundary conditions are set as the Neumann boundary condition. No slip boundary condition has been used at the wall. Wall functions are applied to describe the turbulent flow properties in the near wall reason.

The initial conditions for particle tracking include the starting position and initial velocities of particles. For this study, the particles are assumed as skin flakes generated from the foreheads of the surgical staff members having the densityof $\rho_{\mathrm{P}}=850 \mathrm{~kg} / \mathrm{m}^{3}$. Size of particle is selected as 15 microns diameter [14]. The representative number of particles generated is 512 for 1 hour of surgery [14]. Other boundary conditions regarding the rate of generation of contaminant particles, heat generations from equipment and human bodies havebeen illustrated in Fig. 4.

When particles reach air supply inlets or exhaust outlets, they will escape and the trajectories terminate. When reaching a rigid object, particles may either attach to or rebound from the object's surface. Particles in a ventilated room are most likely to attach to the surface since they usually cannot accumulate enough rebound energy to overcome adhesion [15]. It is therefore natural to terminate, or "trap", a particle trajectory after hitting a rigid surface. This treatment was adopted and used in many CFD studies of the indoor environment.

The trap treatment, however, is not suitable for the current situation, which uses a high Reynolds number k- $\varepsilon$ model. Instead of using trap treatment, this study set the restitution coefficient to a very small value. By doing so, particles were immediately stopped without being trapped after reaching a surface. When particles acquired sufficient normal velocity, they escaped from the boundary layer and became re-suspended. This implies that deposition is neglected. Such manipulation may only be suitable when particle deposition rate is very low.

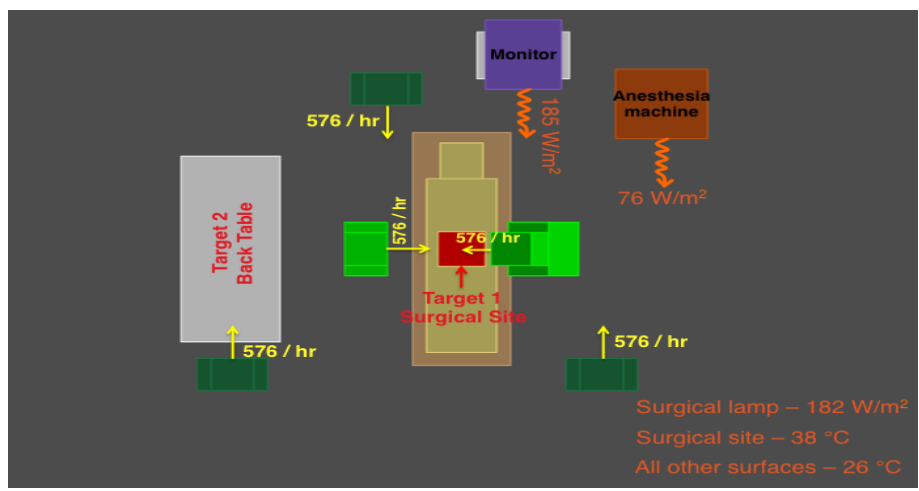

Fig. 4 Boundary Conditions

\section{Modeling procedures}

The CFD solver FLUENT 6.0 [16] has been used to numerically solve the Navier-Stokes equations. This solver has a finite volume solution scheme for the mean momentum, energy, and turbulent transport equations.As part of the same package, a pre-processor GAMBITis used to generate the required grid for the solver. An unstructured grid with rectangular elements is used. The whole surgical side domain has been divided into 629762 equally spaced elements as shown in figure 5 . The turbulence model used in this work is based on a two-equation model (i.e., a standard k- $\varepsilon$ model from Launder and Spanlding [11]). Incompressible flow is assumed; thus the use of a segregated solver is adequate. The schemes selected to perform computations is "second-order upwind implicit". The SIMPLE method is selected to solve the pressure-velocity coupling. 
Particle tracking for turbulent dispersion of particles in a turbulent flow is calculated by using stochastic model of FLUENT 6.0. Number of tries of particle tracking was set to 8 to get the representative number of 512 released particles from each source since surface element size was having 64 elements generating each particle.

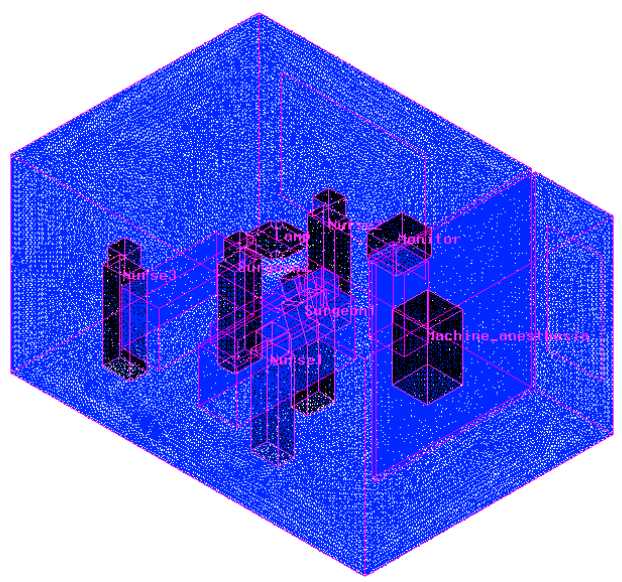

Fig. 5Mesh used for the operating room

\section{Numerical Results}

Figure 6 shows the numerical result of CFD simulation. It shows that SSSE ventilation system provides the shorter path length across the occupied area. This will effectively isolate patient from the operating staff and dilute the contaminated particles to escape from the operating room.

In particles trajectory perspective, $87.8 \%$ particles generated from surgeons and $95.1 \%$ particles generated from nurses have been escaped from the operating room out of 512 representative numbers of particles. This system exhibits very good performance in terms of number of particles strike on wound area (i.e. no particles strikes on wound area).

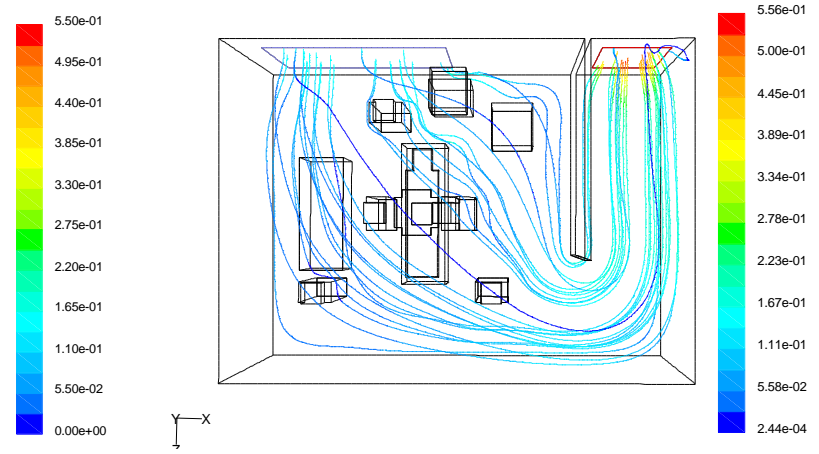

(a) Streamlines of airflow

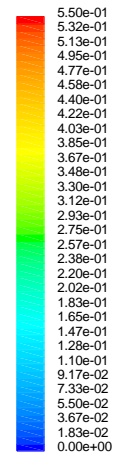

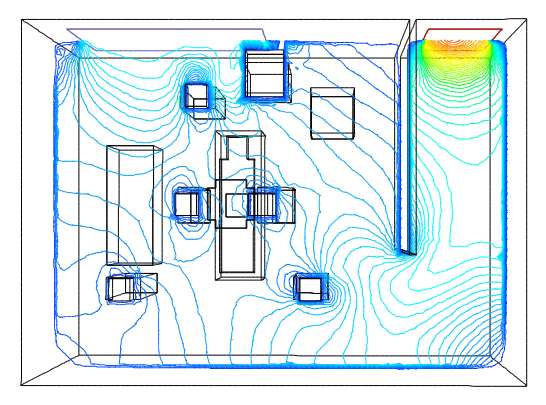

$F^{-x}$

(c) Velocity contours

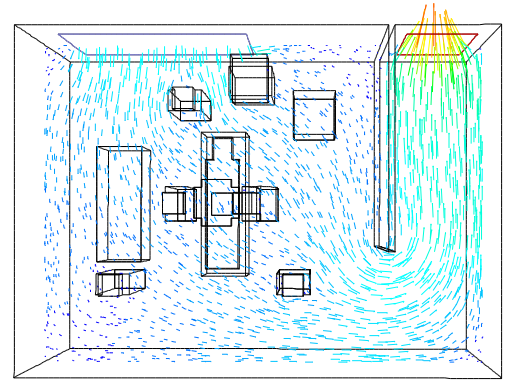

$T^{-x}$

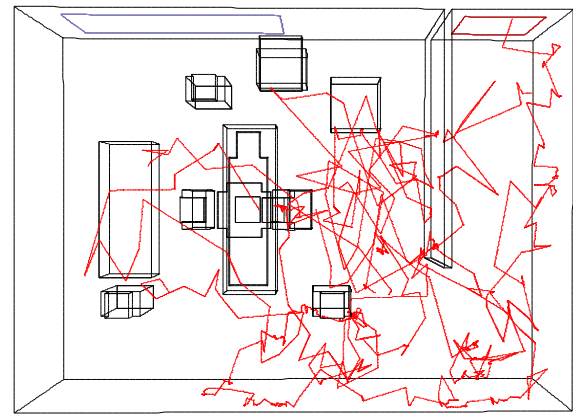

(d) Particle trajectory

Fig. 6 Numerical Simulation of SSSE system (Top view) 


\section{Conclusion}

Horizontal airflow pattern can provide an important alternative to operating room ventilation airflow design. When the air supply and exhaust facilities are installed on the same lateral wall to generate a horizontal unidirectional flow ventilation in the operating room, the medical lamp and thermal plume induced by temperature difference between people and environment have no obvious influence on the airflow pattern of the occupant area, and the airflow pattern is easy to keep unidirectional. This system performs well in avoiding the influence of the surgeons to the patient. Particles released by surgeons can be wiped off effectively, without leading to the increase of the particle concentration surroundingthe patient. The risk of the post-operative infection can thus be decreased significantly. The relative position of source and layout of the operating room highly influence the particle concentration surrounding the patient. Low particle concentration surrounding the patient, especially around the wound area can be maintained by prescribing the direction of the patient correctly, according to the character of the surgical. The main principle to set the layout is to make sure that the main source of particle is in a downstream location of the wound area.

\section{REFERENCES}

[1] Health care facilities. In: ASHRAE handbook. American Society of Heating, Refrigerating and Air-conditioning Engineers; 1999 [chapter 7].

[2] Humphreys H. Infection control and the design of a new operating theatre suite. The Journal of Hospital Infection 1993;23(1):6170.

[3] Sehulster L, Chinn R, Guidelines for environmental infection control in health- care facilities : Recommendations of CDC and the Healthcare Infection Control Practices Advisory Committee (HICPAC). Morbidity and Mortality Weekly Report 2003; 52(10):142. Available at http://www.cdc.gov/mmwr/preview/mmwrhtml/rr5210a1.htm.

[4] Monika W, Joseph V, Ste phane M. Diagonal air-distribution system for operating rooms: experiment and modeling. Building and Environment2004;39(10):1171-8.

[5] Ling J, Study on ventilation system of operating room, School of EnvironmentScience and Technology, Tianjin University; 2005 [in Chinese].

[6] Chow TT\&Yang XY, Ventilation performance in the operating theatre against airborne infection: Numerical study on an ultra-clean system. Journal of Hospital Infection 2005;59:138-147.

[7] Me' ndez C, Jose' JFS, Villafruela J M, Castro F, Optimization of a hospital room by means of CFD for more efficient ventilation, Energy and Buildings, 2007;40(5):849-854.

[8] Chow TT, Yang XY, Performance of ventilation system in a non-standard operating room, Building and Environment, 2003;38(12):1401-1411.

[9] Junjie Liu*, Haidong Wang \&Wenyong Wen, Numerical simulation on a horizontal airflow for airborne particles control in hospital operating room, Building and Environment, 44, (2009), 2284-2289.

[10] Patankar, S V, Numerical Heat Transfer and Fluid Flow, McGraw Hill, Washington, (1980).

[11] Launder, B E, Spalding, D B, 1972, Lectures in Mathematical Models of Turbulence. Academic Press, London, England.

[12] Sinha, S L, Arora, R C and Subhransu Roy, Numerical Simulation of Two Dimensional Room Air Flow with and Without Buoyancy, Energy And Buildings, 32(1), pp. 121-129 (2000).

[13] Press, W H, Teukolsky, S A, Vetterling W T and Flannary B P, Numerical recipes in FORTRAN, Second edition, Cambridge, Cambridge University Press, (1992).

[14] FarhadMemarzadeh, Andrew P. Manning, Comparison of Operating Room Ventilation Systems in the Protection of the Surgical Site.

[15] Hinds W C, Aerosol Technology, Properties, Behavior, and Measurement of Airborne Particles. Wiley, New York, (1982).

[16] FLUENT, Fluent 6.0 User's guide. Fluent Inc., Lebanon, NH. 